\section{letters}

\section{TO THE EDITOR}

Please submit letters for the

Editor's consideration within three weeks of receipt of the Journal.

Letters should ideally be limited to 350 words, and can be submitted on disk or sent by email to:

Clinicalmedicine@rcplondon.ac.uk.

\section{Poisons: initial assessment and management}

Editor - We very much enjoyed the paper by Bradberry and Vale (Clin Med March/ April 2003, pp 107-10) but would like to point out that whilst intravenous flumazenil is considered relatively safe when used with iatrogenic benzodiazepine overdose, it is dangerous when given to patients in A\&E whom you suspect or know to have taken benzodiazepines. This is because it may result in seizures which do not respond to conventional first-line therapy.

JOHN TRENFIELD

Specialist Registrar in Emergency Medicine FEY PROBST

Consultant in Emergency Medicine

Charing Cross Hospital, London

Editor - Bradberry and Vale (Clin Med March/April 2003, pp 107-10) are correct to advise a 'systematic approach to the poisoned patient'. However, it would be remiss not to raise related issues that, if overlooked, would be detrimental to patient care. They state that initial assessment of the patient's state is based on 'the established clinical method of history, examination and investigation.' We do not dispute the importance of this but suggest that initial assessment should begin with 'A (airway), B (breathing), C (circulation) and D (Disability)'.

Our argument is based on the premiss that a low or falling score on the Glasgow Coma Scale (GCS) is associated with air- way obstruction (partial or complete), diminished upper airway reflexes and subsequent risk of aspiration. ${ }^{1}$ Failure to recognise signs of respiratory compromise and of clouded consciousness have been implicated in poor outcome. ${ }^{2,3}$ Without early protection of the airway (endotracheal intubation), respiratory morbidity increased in severe acute poisoning patients. ${ }^{4}$ This resulted in an increased incidence of aspiration, prolonging intubation times and length of stay on intensive care. Guidelines on the management of the acutely poisoned were subsequently amended.

In our NHS Trust, $10-15 \%$ of acute poisoning patients have impaired conscious levels on initial assessment by ambulance personnel (Newcastle Hospitals NHS Trust Audit Department, personal communication). We therefore recommend that clinicians have a high index of suspicion in relation to airway and ventilatory compromise in such patient groups. Guidelines for intubation and ventilation in the unconscious patient may be helpful (see Table 1).

Bradberry and Vale offer an in-depth review of the investigative management of acutely poisoned patients. However, their description of the initial assessment of such patients is sketchy and should include ventilatory, circulatory and GCS assessment to facilitate early recognition of problems.

\section{References}

1 Rello J, Diaz E, Roque M, Valles J. Risk factors for developing pneumonia within 48 hours of intubation. Am J Resp Crit Care Med 1999;159(6):1742-6.
2 Sax FL, Charlson ME. Medical patients at high risk of catastrophic deterioration. Crit Care Med 1987;15:510-15.

3 McQuillan P, Pilkington S, Allan A, Taylor $\mathrm{B}$ et al. Confidential enquiry into quality of care before admission to intensive care. BMJ 1998;316:1853-8.

4 Cosgrove JF, Gascoigne AD. Inadequate assessment of the airway and ventilation in acute poisoning. A need for improved education. Resuscitation 1999;40:161-4.

5 Gentelman et al. BMJ 1993;307:547-52.

JOSEPH F COSGROVE Consultant in Anaesthesia and Critical Care Freeman Hospital, Newcastle upon Tyne

ALISTAIR D GASCOIGNE

Consultant in Respiratory Medicine and Critical Care

Royal Victoria Infirmary, Newcastle upon Tyne

\section{Consent with understanding: a movement towards informed decisions - how far do we go?}

Editor - Mayberry and Mayberry's contribution to understanding and obtaining consent has always been illuminating, in particular the concept of moving away from passive informed consent towards active informed decisions by patients (Clin Med November/December 2002, pp 523-6). The quality of information provided and the way it is understood is vital. Alternatives should be discussed, together with associated risks and complications. Mayberry and Mayberry have also shown, in a study of information leaflets used in endoscopy units, that clinical negligence solicitors felt consent should be obtained at least two weeks before the procedure and patients told about the test

Table 1. Indications for intubation and ventilation of coma patients (from Ref 5).

\title{
Immediate indications
}

- Coma: not obeying commands, not speaking, not eye opening, ie: GCS < 8

- Loss of protective laryngeal reflexes

- Ventilatory insufficiency as judged by arterial blood gases: hypoxaemia ( $\mathrm{Pa} O 2<9 \mathrm{kPa}$ on air or $<13 \mathrm{kPa}$ on oxygen) hypercarbia ( $\mathrm{Pa} \mathrm{CO} 2>6 \mathrm{kPa})$

- Spontaneous hyperventilation causing $\mathrm{PaCO} 2<3.5 \mathrm{kPa}$

- Respiratory arrhythmia

Indications before transferring a patient (including within a hospital)

- Significantly deteriorating conscious level, even if not coma

- Bilateral fractured mandible

- Copious bleeding into mouth

- Seizures 
on at least two occasions. ${ }^{1}$ Fewer patients, however, expressed a similar view.

This whole process would naturally involve a substantial amount of time and highlights the fact that obtaining consent is not an isolated event but rather a continuing dialogue.

Their study also raises other issues, particularly when obtaining consent in the arena of endoscopy, and the practicalities of doing so in a busy and demanding NHS practice. When discussing risks, how much is enough? Mayberry's study would suggest that $16 \%$ of clinical negligence solicitors and only $6 \%$ of patients would like to be informed of risks up to one in a million. ${ }^{1}$ It is worth noting here that the risk of sustaining a serious injury while driving a car is only 1 in 5,000. Perhaps we should adapt the view of our colleagues across the water and discuss every risk no matter how small. There is still a problem of where to draw the line. The recent National Colonoscopy Audit carried out jointly by the British Society of Gastroenterology, the Royal College of Physicians and the Royal College of Surgeons has shown, for example, the perforation rate for a diagnostic colonoscopy was $1: 1,000$ and that for a therapeutic procedure 1:500. The consequences of a perforation may involve a laparotomy and perhaps a stoma. Should we mention this at the outset when obtaining consent?

What should we tell patients about who performs the procedure? In an accredited endoscopy unit there will usually be a trainee and, in most units now, a trained nurse endoscopist. Should the patient be given the choice as to who performs the procedure? Mayberry has also alluded to performance statistics and local disclosure panels. Thus, the patient probably has the right to know what the individual endoscopist's terminal ileal intubation rates and complication rates are, for example; or should they only be disclosed when asked specifically? The implications of this are enormous, needless to say. Furthermore, with the advent of the Cancer Plan and the two-week wait, there has been a surge in the workload and hence the formation of a one-stop colorectal clinic. Here the patient is assessed clinically and then a flexible sigmoidoscopy is performed followed by a barium enema on the same day. In such cases, patients do not have time to contemplate the procedures beforehand and physicians cannot assess recall or understanding.

Finally, who should actually obtain consent? Should it be the physician/surgeon performing the procedure itself or the individual initiating the test? Often they are not the same. Or perhaps it should be nurse specialists, in their ever evolving role?

I believe wholeheartedly in the principle of involving the patient from the outset of the consultation and moving towards informed decisions, but we have still far to

Clinical \& Scientific letters go in putting this into practice. If patient care is to be improved, these issues should be addressed within the individual departments and trusts.

\section{References}

1 MK Mayberry, JF Mayberry. Towards better informed consent in endoscopy: a study of information and consent process in gastroscopy and flexible sigmoidoscopy. Eur J Gastroenterol Hepatol 2001:13:1-10.

RP ARASARADNAM Specialist Registrar Gastroenterology/Medicine Sheffield Teaching Hospitals, Sheffield

\section{Letters not directly related to articles published in Clinical Medicine and presenting unpublished original data should be submitted for publication in this section. Clinical and scientific letters should not exceed 500 words and may include one table and up to five references.}

\section{Rate control in atrial fibrillation}

Atrial fibrillation (AF) is the most common sustained tachyarrhythmia encountered in clinical practice. ${ }^{1}$ Its prevalence increases with age and it is associated with a doubling of overall morbidity and mortality from cardiovascular disease. ${ }^{1}$

Three strategies form the basis of AF management: restoration and maintenance of sinus rhythm, rate control and prevention of stroke. Randomised trials have provided strong evidence of a substantial reduction in stroke with warfarin (target INR of 2.0-3.0). ${ }^{1}$ Clinical factors associated with an increased risk for stroke include age $\geq 65$ years, congestive cardiac failure, diabetes mellitus, previous stroke or transient ischaemic attack and hypertension. ${ }^{1}$ For patients in persistent AF, until recently there were few clinical data available directly comparing a rate versus rhythm control strategy.

However, in four recent trials (PIAF, ${ }^{2}$ RACE, ${ }^{3}$ AFFIRM, ${ }^{4}$ and STAF-Pilot ${ }^{5}$ ) patients in persistent AF were randomly allocated to rate versus rhythm control treatment. The results provide compelling evidence that rate control is as good as a rhythm control strategy in terms of improvement in symptoms, quality of life, and mortality, and may be an acceptable and preferred first-line treatment for the majority of patients with persistent AF, who have a high risk of recurrence. Several other important messages emerged from these trials:

- the importance of maintaining longterm therapeutic anticoagulation even if sinus rhythm is quickly restored

- the poor efficacy of current rhythm control strategies, with a high crossover rate from the rhythm to rate control strategy

- rhythm control strategies are associated with higher hospitalisation rates and more frequent adverse drug effects.

Having accepted that rate control is the appropriate strategy for most persistent AF patients, clinicians need clear guidance on what is considered acceptable ventricular rate control. Control of the resting heart rate does not automatically imply adequate heart rate regulation during exercise. The American College of Cardiology/American Heart Association/ European Society of 